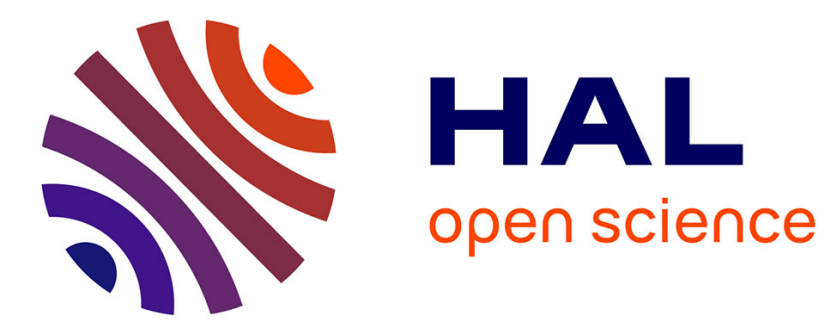

\title{
D'Alembert function for exact non-smooth modal analysis of the bar in unilateral contact
}

David Urman, Mathias Legrand, Stéphane Junca

\section{To cite this version:}

David Urman, Mathias Legrand, Stéphane Junca. D'Alembert function for exact non-smooth modal analysis of the bar in unilateral contact. Nonlinear Analysis: Hybrid Systems, 2021, 10.1016/j.nahs.2021.101115 . hal-02984137v2

\section{HAL Id: hal-02984137 \\ https://hal.science/hal-02984137v2}

Submitted on 17 Nov 2021

HAL is a multi-disciplinary open access archive for the deposit and dissemination of scientific research documents, whether they are published or not. The documents may come from teaching and research institutions in France or abroad, or from public or private research centers.
L'archive ouverte pluridisciplinaire HAL, est destinée au dépôt et à la diffusion de documents scientifiques de niveau recherche, publiés ou non, émanant des établissements d'enseignement et de recherche français ou étrangers, des laboratoires publics ou privés.

\section{다(1) $\$$}

Distributed under a Creative Commons Attribution - NonCommercial| 4.0 International 


\title{
D'Alembert function for exact non-smooth modal analysis of the bar in unilateral contact
}

\author{
David Urman ${ }^{1}$, Mathias Legrand ${ }^{1}$, and Stéphane Junca ${ }^{2}$ \\ ${ }^{1}$ Department of Mechanical Engineering, McGill University, Montreal, Canada \\ ${ }^{2}$ LJAD Mathematics, Inria \& CNRS, Université Côte d'Azur, Nice, France
}

November 17, 2021

\begin{abstract}
Non-smooth modal analysis is an extension of modal analysis to non-smooth systems, prone to unilateral contact conditions for instance. The problem of a one-dimensional bar subject to unilateral contact on its boundary has been previously investigated numerically and the corresponding spectrum of vibration could be partially explored. In the present work, the non-smooth modal analysis of the above system is reformulated as a set of functional equations through the use of both d'Alembert solution to the wave equation and the method of steps for Neutral Delay Differential Equations. The system features a strong internal resonance condition and it is established that irrational and rational periods of vibration should be carefully distinguished. For irrational periods, it was previously proven that the displacement field of the non-smooth modes of vibration is characterized with piecewise-linear functions in space and time and such a motion is unique for a prescribed energy. However, for rational periods, which are the subject of this work, new periodic solutions are found analytically. Findings consist of families of iso-periodic solutions with piecewise-smooth displacement fields in space and time and continua of piecewise-smooth periodic solutions of the same energy and frequency.
\end{abstract}

Keywords: unilateral contact, non-smooth modal analysis, periodic solutions, vibration analysis, Signorini complementarity conditions, d'Alembert function, method of steps, Neutral Delay Differential Equation

\section{Contents}

1 Introduction $\quad 2$

2 Problem Statement 2

3 D'Alembert Solution to the Signorini Problem 4

3.1 Important Terminology 4

3.2 Considered Solution Space 4

3.3 Solution via the method of steps 5

3.3.1 Inactive Contact Motion 6

$\begin{array}{lll}\text { 3.3.2 Active contact motion } & 8\end{array}$

$\begin{array}{ll}\text { 3.3.3 Periodicity and Admissibility conditions } & 9\end{array}$

3.3.4 Summary of necessary conditions for periodic solutions 11

4 Non-smooth modal analysis $\quad 12$

4.1 Known properties of the non-smooth modal space 12

$\begin{array}{llr}4.2 & \text { Piecewise-linear mode } & 13\end{array}$

4.3 Piecewise-smooth mode(s) of the same period 16

4.4 Applications to non-smooth modal analysis 19

5 Conclusion $\quad 21$ 


\section{Introduction}

Non-smooth modal analysis (NSA) is used to obtain resonant vibratory responses and frequencies for structures prone to unilateral contact conditions [6,19]. While linear modes of vibration are obtained by solving eigenvalue problems, non-smooth modes are seen as invariant manifolds supporting periodic autonomous solutions [17].

The NSA of a simple one-dimensional cantilever bar has recently attracted experimental interest [13] and has already been investigated analytically and numerically in semi-discrete and continuous settings in space $[1,9,10,16,18-20,23]$. In the continuous framework, modes consisting of piecewiselinear solutions in space and time have been shown to exist $[19,22,23]$. Similarly to nonlinear modes in semi-discrete systems commonly reported in the mechanical engineering literature [7,12], these modes were found to exist for continua of frequencies and energy magnitudes $[1,19,22]$. Such continua are widely referred to as backbone curves. Previous research has also concluded that some of the nonsmooth modes feature purely vertical backbone curves, with periodic motions of the same period but different energies [20].

The present paper revisits and extends existing results through new theorems on the structure of the modal space together with the construction of new non-smooth modal families. The above system enjoys the well-known d'Alembert travelling-wave solution and is known to be mathematically wellposed without the use of an impact law. It also features a strong internal resonance condition since all linear eigen-frequencies of the clamped-free bar system are multiples of the first one. Such properties make it ideal for NSA without prior classical semi-discretization in space such as the Finite Element Method. Specifically, the investigated Signorini problem is ill-posed in the framework of Finite Element method and is often complemented with additional conditions [3]. For example, some schemes may incorporate a Newton impact law [2] or a penalization term [21]. Penalization techniques, of the type presented in $[3,11,21]$, require that a penalization parameter reach infinity to guarantee no penetration of the obstacle. Thus, these are not used as they do not depict accurately the Signorini conditions. On the other hand, application of a Newton impact law does satisfy the Signorini conditions in the finite element framework (in continuous time or as the time step reaches zero [2]). A continuum of periodic solution could be found by implementing a purely elastic impact law which allows for energy conservation [9] (other Newton impact laws dissipate energy and therefore annihilate periodic solutions). However, under the purely elastic impact law, the bar exhibits a chattering behaviour at the contact interface which does not exist in the exact solution of the investigated system [3].

The outline is as follows: the formulation of the bar in unilateral contact is briefly recalled in section 2. Preliminaries to NSA, such as d'Alembert's solution to the contact problem, formulation of the periodicity condition, and lemmas on the existence of solutions are exposed in section 3. Finally, NSA is performed and compared with existing literature in section 4.

\section{Problem Statement}

The formulation of interest has already been established $[22,23]$ and is briefly recalled for the purpose of context and introduction to appropriate terminology for the remainder of the article.

As depicted in Figure 1, we consider the displacement field $u(\tilde{x}, \tilde{t})$ inside a bar of length $L$, where $\tilde{x}$ and $\tilde{t}$ denote physical position and time, respectively. Assuming linear elasticity, the displacement

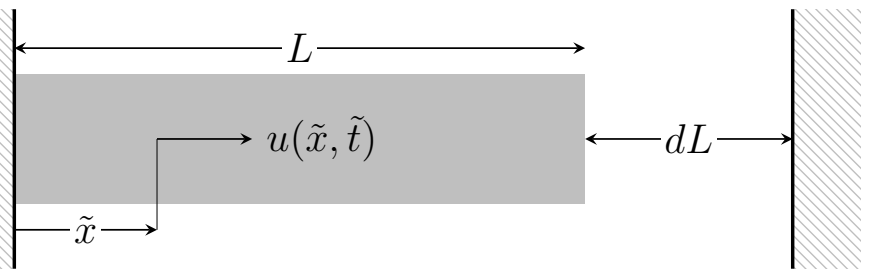

Figure 1: Unilaterally constrained cantilever bar. 
of the bar satisfies the non-dimensional wave equation

$$
u_{t t}(x, t)=u_{x x}(x, t), \quad x=\tilde{x} / L, \quad t=c \tilde{t} / L,
$$

where $c=\sqrt{\rho / Y}>0$ is the speed of sound in the bar ( $\rho$ denotes the density of the bar and $Y$, its Young modulus) and subscript denotes partial differentiation with respect to the denoted variable. To simplify further the notations, the dimensionless coordinates $x$ and $t$ are introduced and, for the remainder of the article, the displacement is expressed exclusively in these coordinates, i.e. $u(x, t)$. As non-smooth modal analysis requires obtaining periodic solutions of the autonomous system $[7,17,19]$, the analysis will be performed on a single period of motion, of normalized period $T$ such that the domains $t \in[0, T]$ and $x \in[0,1]$ are considered.

On the boundary $x=1$, the bar is prone to unilateral contact with a rigid wall initially at a normalized distance $d>0$. The contact conditions are treated using the Signorini complementarity conditions stating that there exist two mutually exclusive phases: the bar can either move freely without contacting the obstacle (inactive contact) or contact the wall while experiencing a repulsive force (active contact) [21-23]. For the sake of simplicity, non-smooth modes consisting of a single active contact per period are sought; these are referred to as $1 \mathrm{CPP}$ (one active Contact Phase per Period) modes [19,22].

Definition 1 (1CPP motion). A periodic motion of the bar with period $T$ is said to be a $1 C P P$ motion if there exist an instance in time $t_{0}$ and a duration $\tau<T$ such that the bar is in inactive contact for $t \in\left[t_{0}, t_{0}+\tau\right]$ and in active contact for $t \in\left[t_{0}+\tau, t_{0}+T\right]$.

Without loss of generality, we can pick $t_{0}=0$. A $1 \mathrm{CPP}$ motion at $x=1$ with $t_{0}=0$ is illustrated in Figure 2.

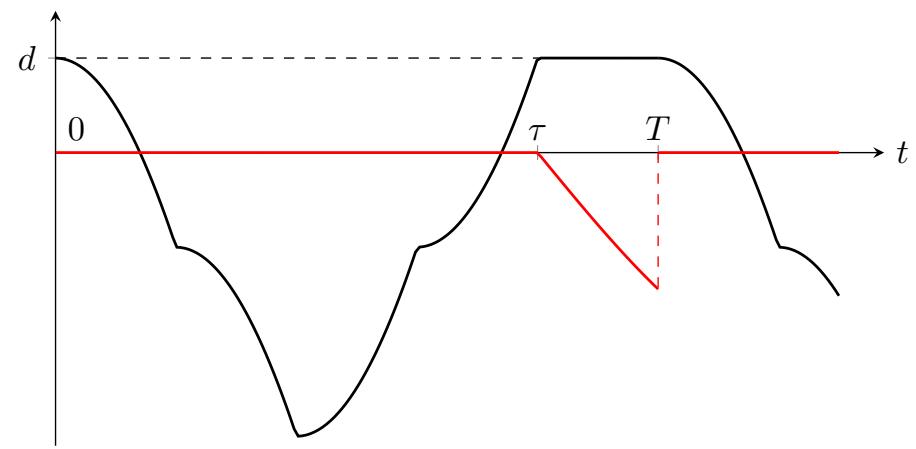

Figure 2: Example of a 1CPP motion: $u(1, t)[-]$ and $u_{x}(1, t)[-]$.

A 1CPP motion should satisfy the boundary conditions

Cantilever bar $\quad u(0, t)=0 \quad \forall t \in[0, T]$

Inactive contact $\quad u(1, t) \leq d \quad \forall t \in[0, T] \quad$ and $\quad u_{x}(1, t)=0 \quad t \in[0, \tau]$ a.e.

Active contact $\quad u(1, t)=d \quad \forall t \in[0, T] \quad$ and $\quad u_{x}(1, t) \leq 0 \quad t \in[\tau, T]$ a.e.

together with the periodicity conditions

$$
\begin{aligned}
u(x, 0) & =u(x, T) \quad \forall x \in[0,1] \\
u_{t}(x, 0) & =u_{t}(x, T) \quad x \in[0,1] \text { a.e. }
\end{aligned}
$$

where a.e. denotes almost-everywhere in the prescribed domain. The indication a.e. shall apply to constraints involving the derivatives of the displacement field (stress and velocity) due to the choice of solution space, further discussed in section 3.2.

Note that solutions where the bar enters into contact with the wall with zero incoming velocity, known as grazing motion [2], are valid solutions to (1)-(4). However, such solutions are intentionally 
omitted in the discussion for sake of conciseness. Accordingly, the restriction where the tip of the bar must approach the wall with positive incoming velocity just before contact is considered:

$$
\lim _{t \rightarrow \tau^{-}} u_{t}(1, t)>0 .
$$

Conditions (3) and (4) generate non-smooth displacement and velocity fields. Since the displacement is expected to be continuous from a physical perspective, continuous weak solutions of the wave Equation (1) are sought. As a consequence, its derivatives in (3)-(6) will only be defined almost everywhere.

\section{D'Alembert Solution to the Signorini Problem}

The d'Alembert solution satisfying Equations (1) and (2) reads [5, p. 69]

$$
u(x, t)=f(t+x)-f(t-x), \quad \forall(x, t) \in[0,1] \times[0, T] .
$$

Note that the function $f$, referred to as the d'Alembert function, can be defined up to a constant. In the sequel, the convention

$$
f(-1)=0
$$

is chosen and does not affect the displacement, velocity or stress solutions derived in this paper. With $t=0$ and $x=1$ in (8), this convention yields $f(1)=u(1,0)$. Moreover, for $(x, t) \in[0,1] \times[0, T]$, $f$ has to be defined on the interval $[-1,1+T]$. Defining $f$ is, therefore, equivalent to determining a cantilever motion of the bar. Section 3 shall hence show how the boundary conditions (3)-(6) are translated into conditions on the d'Alembert function.

In previous research on the topic of interest $[19,22,23]$, NSA was formulated using the displacement, stress and velocity fields and was performed by computing initial conditions that generate admissible periodic solutions. Instead, in the present article, the d'Alembert function is exploited exclusively, which leads to new insights. To illustrate the idea, a known admissible periodic solution and its associated d'Alembert function are plotted in Figure 3.

Before solving Equations (1)-(6) using the d'Alembert function, the considered solution space is characterized.

\subsection{Important Terminology}

In this paper, we define non-smooth modes as families of motions defined by non-smooth d'Alembert functions. Piecewise-linear modes consist of piecewise-linear d'Alembert solutions, as depicted in Figure 3 and piecewise-smooth modes consist of piecewise-smooth d'Alembert functions, which include piecewise-linear d'Alembert functions. This distinction will be used to further emphasize the novelty presented in this manuscript. To clarify, an example of a piecewise-smooth yet not piecewise-linear d'Alembert function is illustrated later in the article, in Figure 11, where the newly detected piecewisesmooth modes are discussed. Still, it should be understood that both families (piecewise-smooth and piecewise-linear) pertain to the family of non-smooth modes.

\subsection{Considered Solution Space}

Due to the Signorini condition, smooth solutions $u$ are not expected. Instead, it is decided to construct solutions with a continuous $\mathcal{C}^{0}$ and piecewise- $\mathcal{C}^{1}$ (denoted $\hat{\mathcal{C}}^{1}$ ) displacement field. This stems from (a) the fact that the displacement should be a continuous function because a mechanical failure of the bar is not of interest, and (b) the fact that the stress $u_{x}$ on the boundary $x=1$ should be defined almost everywhere. Thus, at each instance in time $t$, there is only a finite number of discontinuities in $x$ for the derivatives $u_{x}(\cdot, t)$ and $u_{t}(\cdot, t)$, where right and left derivatives exist but differ. Such assumptions are sufficient to cover all presented solutions and methodology in this paper. Moreover, this solution space consists of valid weak solutions to the wave equation [5, p. 407-408]. 


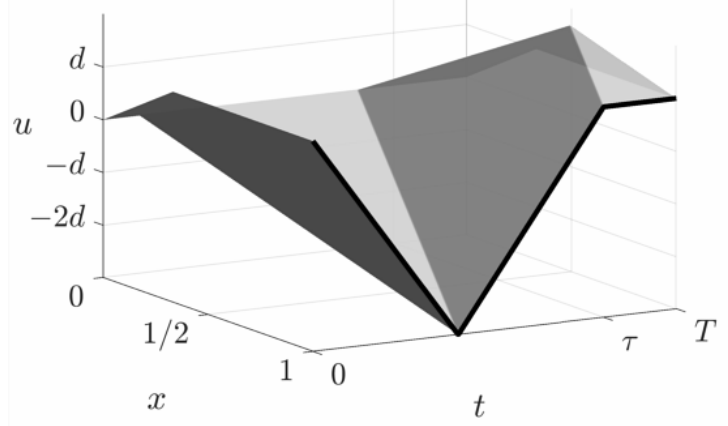

(a) Displacement field $u(x, t)=f(t+x)-f(t-x)$ with motion of tip of the bar $u(1, t)[-]$.

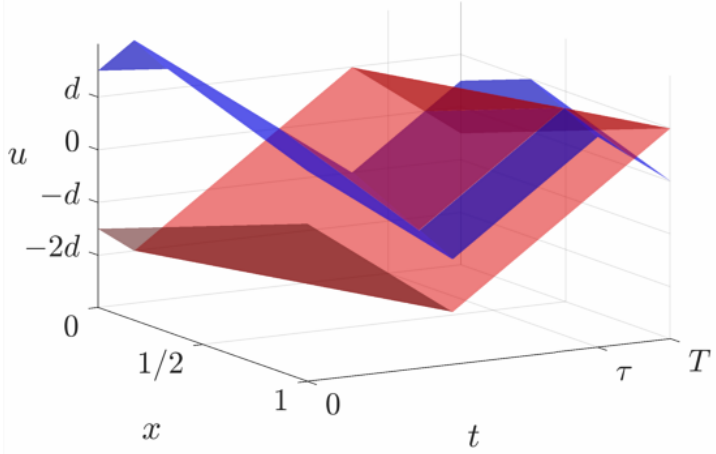

(b) d'Alembert functions from Figure 3(a) seen as surfaces: $u(x, t)=f(t+x)[\square]$ and $u(x, t)=f(t-$ $x)[\square$.

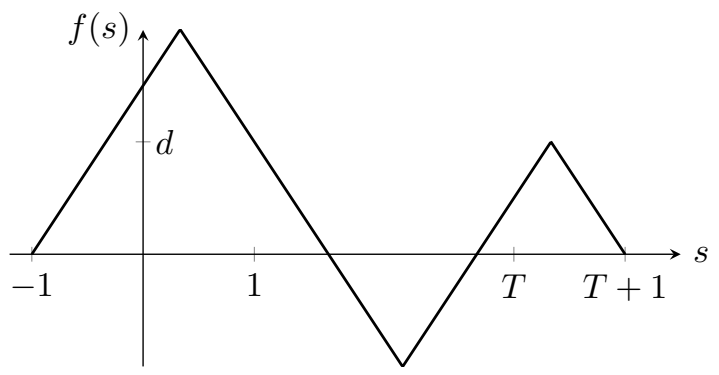

(c) (Piecewise-linear) d'Alembert function $f(s)$ corresponding to Figure 3(a).

Figure 3: A 1CPP motion [23] and corresponding d'Alembert function

Accordingly, $f$ is chosen as a continuous and piecewise- $\mathcal{C}^{1}$ function on its domain of definition, that is $f \in \mathcal{C}^{0}([-1,1+T]) \cap \hat{\mathcal{C}}^{1}([-1,1+T])$. This means that the classical derivative of $f$ is defined everywhere except over a finite number of points on any bounded set. Also, at a point $s$ where $f^{\prime}$ is not defined, the right limit $f^{\prime}\left(s^{+}\right)$and the left limit $f^{\prime}\left(s^{-}\right)$still exist. An equality involving $f^{\prime}$ is thus satisfied at all points of the considered interval except at the finite number of points where $f$ is not differentiable. Within the above framework, it should be noted that solution uniqueness is guaranteed in larger solution spaces $\mathcal{H}^{3 / 2}$ and $\mathcal{H}^{1}[8,15]$. This is used in later proofs.

\subsection{Solution via the method of steps}

Consider the Cauchy problem consisting of the governing PDE (1) with boundary conditions (2)-(4) and initial conditions $u(x, 0)=u_{0}(x)$ and $u_{t}(x, 0)=v_{0}(x)$. The periodicity conditions (5)-(6) will be considered later in section 3.3.3. The d'Alembert function is defined on $[-1,1]$ as $[5$, p. 69$]$

$$
2 f_{0}(s) \equiv 2 f(s)= \begin{cases}u_{0}(s)+C_{0}+\int_{0}^{s} v_{0}(\mu) \mathrm{d} \mu & s \in[0,1], \\ -u_{0}(-s)+C_{0}-\int_{0}^{-s} v_{0}(\mu) \mathrm{d} \mu & s \in[-1,0]\end{cases}
$$

Our convention (9) implies

$$
C_{0}=u_{0}(1)+\int_{0}^{1} v_{0}(\mu) \mathrm{d} \mu
$$

Note that imposing a value on the constant $C_{0}$ does not affect any of the fields of interest: displacement $u$, velocity $u_{t}$ or stress $u_{x}$. Additionally, the notation $f_{0}$ is introduced to describe the part of $f$ depending exclusively on initial conditions, i.e. $f_{0}(s) \equiv f(s) \forall s \in[-1,1]$ (as discussed later, the d'Alembert function beyond $s>1$ depends on both boundary and initial conditions). Therefore, to simplify the terminology used in the remainder, $f_{0}$ will be referred to as initial conditions. Moreover, 
as the d'Alembert function is assumed to be continuous for $s \in[-1,1]$, the space of initial conditions $u_{0}$ and $v_{0}$ consists of those generating piecewise- $\mathcal{C}^{1}$ continuous functions $f_{0}$.

As mentioned previously, the d'Alembert function in (8) is a solution to (1) and (2). However, the boundary condition at $x=1$ remains unsatisfied. To satisfy boundary conditions at both $x=1$ and $x=-1$, the d'Alembert function is generally extended from $[-1,1]$ to $[-1, T+1]$ via initial conditions using reflections at $s=1$ and $s=-1[5,14]$. In the present article, this extension will be derived by solving the boundary conditions Equations (3) and (4) in terms of the d'Alembert function (omitting inequality constraints)

$$
\begin{array}{lll}
\text { Inactive contact } & f^{\prime}(t+1)+f^{\prime}(t-1)=0, & t \in[0, \tau] \text { a.e. } \\
\text { Active contact } & f(t+1)-f(t-1)=d, & t \in[\tau, T] .
\end{array}
$$

Collectively, Equations (12) and (13) represent a degenerate Neutral Delay Differential Equation (NDDE) which requires knowledge of an initial condition on $f$ spanning a domain of length equal to the delay [4]. Namely, to solve (12), the d'Alembert function must be known for any $s \in[-1,1]$ as given by the initial conditions (10). Thus, given $f_{0}$, Equation (12) determines $f$ on $[1, \tau+1]$ and, consecutively, Equation (13) determines $f$ on $[\tau+1, T+1]$.

In what follows, we derive conditions on $f_{0}, \tau$ and $T$ in order to find $1 \mathrm{CPP}$ solutions of the cantilever bar. To this end, we use the method of steps to construct solutions to the inactive contact motion (12) and active contact motion (13) separately using linear arguments. The nonlinearity of the problem at hand arises when the inequalities in (3) and (4) together with periodicity conditions (5) to (6) are enforced on the d'Alembert function.

\subsubsection{Inactive Contact Motion}

This section details the extension of the d'Alembert function to the domain $s \in[-1,1+\tau]$ via boundary condition (12). Beforehand, since (a) the tip of the bar is in contact with the wall at the end of the period, see (4), (b) the motion must be periodic, see (5), and (c) the tip of the bar must be initially in contact with the obstacle to preserve continuity in time from (8), the tip of the bar must contact the obstacle at the beginning of the period

$$
u(1,0)=f(1)-f(-1)=f_{0}(1)-f_{0}(-1)=f_{0}(1)=d .
$$

Next, the inactive contact condition (12) leads to

$$
f^{\prime}(s)=-f^{\prime}(s-2), \quad s \in[1, \tau+1] \text { a.e. }
$$

Integration of (15) reads

$$
f(s)=d-f(s-2), \quad \forall s \in[1, \tau+1] .
$$

By the method of steps, given $f(s)=f_{0}(s)$ for $s \in[-1,1]$, the solution to (16) is

$$
f(s)=\left\{\begin{array}{ll}
f_{0}(s) & s \in[-1,1] \\
d-f_{0}(s-2) & s \in[1, \tau+1]
\end{array}, \quad 0 \leq \tau \leq 2\right.
$$

this definition applies strictly to the case of $\tau \leq 2$ since the domain of definition of initial conditions is of length 2. Next, for $2<\tau \leq 4$, we obtain

$$
f(s)= \begin{cases}f_{0}(s) & s \in[-1,1] \\ d-f_{0}(s-2) & s \in[1,3] \\ d-f(s-2) & s \in[3, \tau+1]\end{cases}
$$

Here, the last step $d-f(s-2) s \in[3, \tau+1]$ is determined by substituting the previous step, $f(s)=$ $d-f_{0}(s-2) s \in[1,3]$, in $f(s-2)$, admitting

$$
f(s)=\left\{\begin{array}{ll}
f_{0}(s) & s \in[-1,1] \\
d-f_{0}(s-2) & s \in[1,3] \\
f_{0}(s-4) & s \in[3, \tau+1]
\end{array}, \quad 2<\tau \leq 4 .\right.
$$




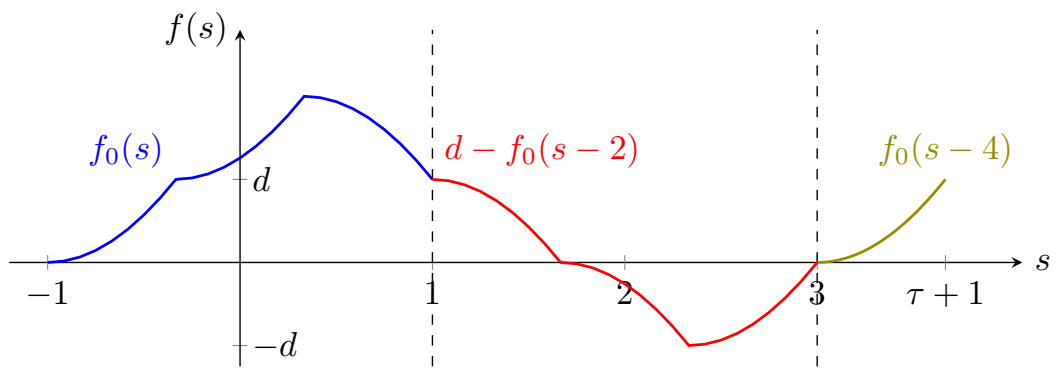

(a) D'Alembert function as defined for $t \in[-1,3]$. Red and Olive functions represent the extension beyond $t=1$, via (19) and depend on the function in blue.

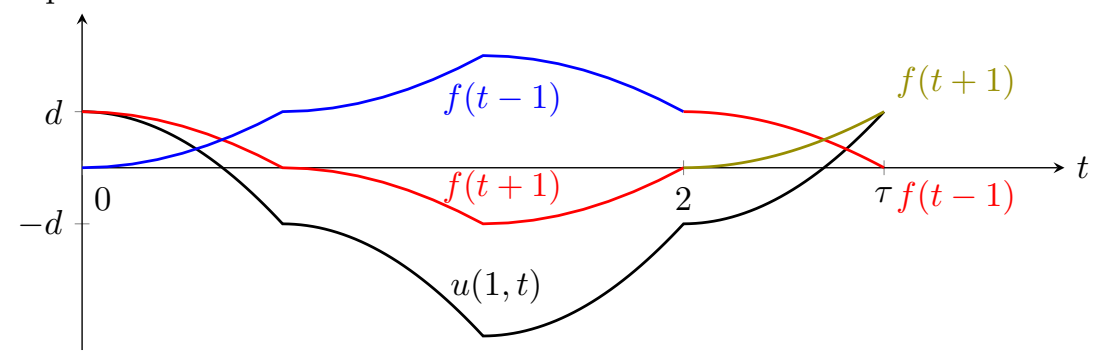

(b) Displacement at tip and d'Alembert function components, $u(1, t)=f(t+1)-f(t-1)$ for $t \in[0,2]$. Colours correspond to plot $4(\mathrm{a})$.

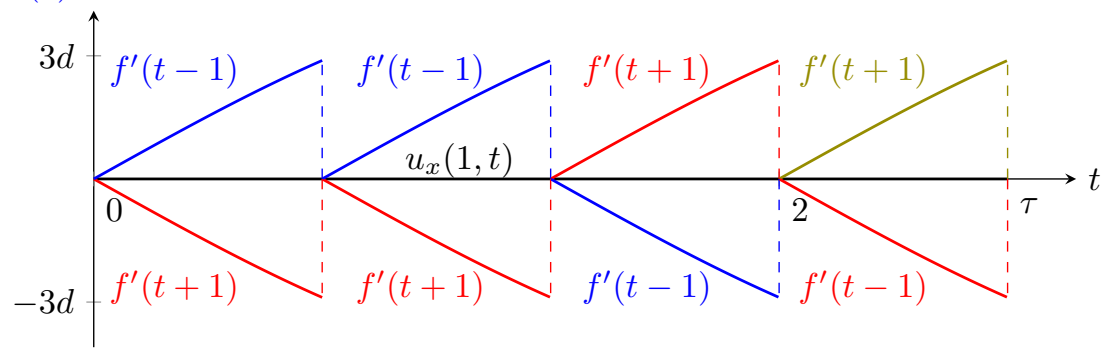

(c) Stress at tip and d'Alembert function components, $u_{x}(1, t)=f^{\prime}(t+1)+f^{\prime}(t-1)=0$ (due to inactive contact), for $t \in[0,2]$. Colours correspond to plot $4(\mathrm{a})$.

Figure 4: Extension of d'Alembert function in inactive contact conditions (15) given specific initial conditions $f_{0}$ (here, piecewise-cubic polynomials for the sake of illustration) and $\tau=7 / 3 \geq 2$, and resulting motion.

A solution of the type (19) is illustrated in Figure 4. Further extension of the d'Alembert function for $\tau>4$ is not illustrated because the d'Alembert function subject to (15) is 4-periodic, that is

$$
f(s)=f(s-4), \quad \forall s \in[3, \tau+1], \quad \tau>2 .
$$

Moreover, we note that there exists an upper bound on the value of $\tau$ for a $1 \mathrm{CPP}$.

Proposition 2 (Maximal duration of an inactive contact for a $1 \mathrm{CPP}$ ). For 1 CPP motions, the duration of inactive contact motion must satisfy

$$
\tau<4
$$

As well, initial conditions must satisfy

$$
\begin{array}{ll}
f_{0}(s) \geq 0 & \forall s \in[-1, \min (1, \tau-1)], \\
f_{0}(s) \leq d \quad \forall s \in[-1, \tau-3] \text { if } 2<\tau<4
\end{array}
$$

Proof. First, we will show that a 1CPP motion cannot occur for $\tau \geq 4$. To this end, we show that a motion with $\tau=4$ will never initiate an active contact phase. Then, by extension, we will show that motions with $\tau>4$ will not initiate an active contact phase either.

To start, we note that a duration $\tau=4$ coincides exactly with the period of the bar if it were always in inactive contact motion (a motion of the bar where $u_{x}(1, t)=0$ for all $t \in[0, \infty)$ ). Thus, a motion 
with $\tau=4$ could hypothetically repeat for $t \in[4, \infty)$ in periods of 4 , as shown in Equation (20), and still satisfy the wave equation (1), cantilever condition (2), and the set of Signorini conditions describing inactive contact motion: $u(1, t) \leq d$ and $u_{x}(1, t)=0$ for all $t \in[0, \infty)$. Specifically, such a motion would be a solution to Signorini problem while never initiating an active contact phase. Using the uniqueness theorem for this Singorini problem [15], we can then justify that a motion that never initiates an active contact phase for $t \in[4, \infty)$ (following the inactive contact motion taking place for $t \in[0, \tau]$ with $\tau=4$ ) is the only possible solution. To clarify, since (I) there must exist a unique solution to the considered initial-value problem [15], and (II) there always exists a solution that is exclusively in inactive contact for $t>\tau=4$; there cannot exist any motion with $\tau=4$ that enters into an active contact phase. By the same logic, we can conclude that any inactive motion that lasts from $t=0$ until some time $t>4$ - for example, a motion with $\tau>4$ - must also be exclusively an inactive contact motion that never initiates active contact. Accordingly, we conclude that a motion with $\tau \geq 4$ never initiates active contact (the stress at $x=1$ is always zero) and, therefore, cannot pertain to the group of $1 \mathrm{CPP}$ motions.

Next, the constraints (22)-(23) are derived by plugging the resulting d'Alembert function for inactive contact into the inactive contact inequality constraint $(3): u(1, t)=f(t+1)-f(t-1) \leq d$ $\forall t \in[0, \tau]$. Starting with the d'Alembert function (17) for $\tau \leq 2$, we obtain

$$
f(t+1)-f(t-1)=d-2 f_{0}(t-1) \leq d \quad \forall t \in[0, \tau], 0<\tau<2
$$

which can be simplified into

$$
f_{0}(t) \geq 0 \quad \forall t \in[-1, \tau-1], 0<\tau \leq 2 .
$$

Next, plugging (17) for $2<\tau<4$ into $f(t+1)-f(t-1) \leq d, \forall t \in[0, \tau]$, we obtain

$$
d \geq f(t+1)-f(t-1)=\left\{\begin{array}{ll}
d-2 f_{0}(t-1) & t \in[0,2] \\
2 f_{0}(t-3)-d & t \in[2, \tau]
\end{array} \quad 2<\tau<4 .\right.
$$

The inequality constraint is then assigned separately in $t \in[0,2]$ and $t \in[2,4]$ and, with further simplification, admits

$$
\begin{array}{llc}
0 \leq f_{0}(t) \quad t \in[-1,1], & 2<\tau<4 \\
d \geq f_{0}(t) & t \in[-1, \tau-3], & 2<\tau<4
\end{array}
$$

At last, conditions (25) and (27) lead to (22), and condition (28) is (23) verbatim which concludes the proof.

In turn, the inactive contact phase ends with a contact initiation, ie

$$
u(1, \tau)=f(\tau+1)-f(\tau-1)=d
$$

and the velocity of the tip at this instant $\tau$ must be strictly positive, due to (7), such that

$$
u_{t}\left(1, \tau^{-}\right)=f^{\prime}\left(\tau^{-}+1\right)-f^{\prime}\left(\tau^{-}-1\right)>0 .
$$

\subsubsection{Active contact motion}

Similarly to the procedure presented in section 3.3.1, the d'Alembert function is extended for $s>\tau+1$ via condition (13), which equivalently reads

$$
f(s)=d+f(s-2), \quad \forall s \in[\tau+1, T+1] .
$$

Note that $f(s-2)$ is assumed to be known for $s \in[\tau+1, T+1]$, by virtue of (17) or (19). An illustration of extension (31) is provided in Figure 5. Moreover, we note that $u_{x}(1, t)$ is 2-periodic during active contact since

$$
u_{x}(1, t+2)=f^{\prime}(t+3)+f^{\prime}(t+1)=f^{\prime}(t+1)+f^{\prime}(t-1)=u_{x}(1, t), \quad t \in[\tau, T] \text { a.e. }
$$

holds true. This sets a bound on the duration $T-\tau$ of active contact. 


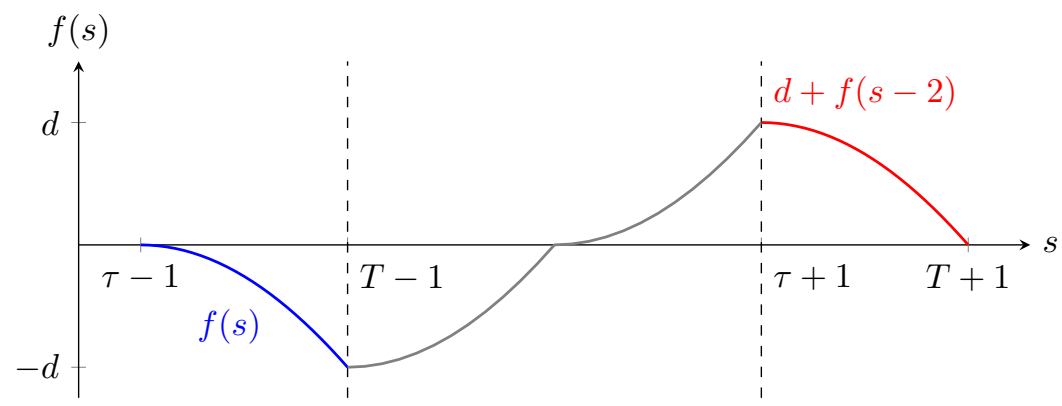

(a) D'Alembert function for $t \in[\tau-1, T+1]$. Extension beyond $t=\tau+1$ via $(31)[-]$ dependent on the blue plot.

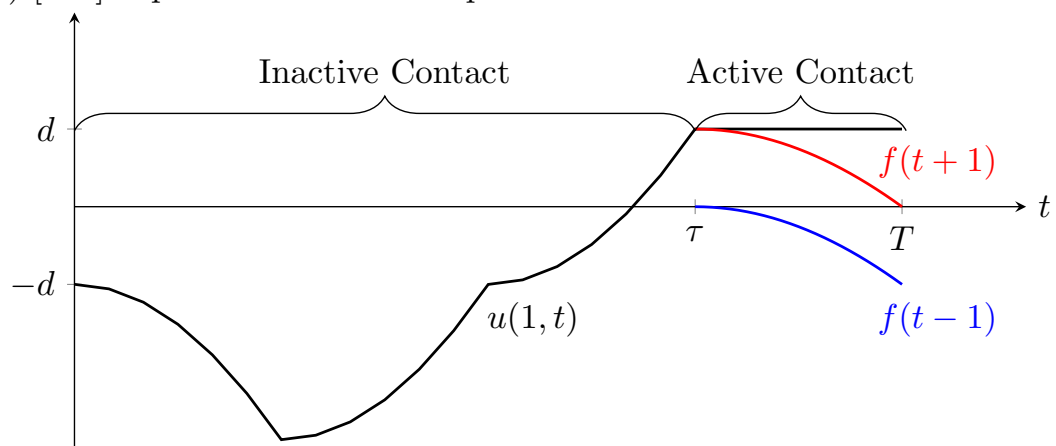

(b) Displacement at tip and d'Alembert function components.

Figure 5: Extension of the d'Alembert function due to active contact conditions (15) given specific initial conditions $f_{0}$ and $f$ in inactive contact motion (shown in Figure 4) from (17) and (19), and resulting motion.

Proposition 3 (Maximal duration of an active contact for a 1CPP). For 1CPP motions, the duration of active contact motion, $T-\tau$ where $T>\tau$, must satisfy

$$
T-\tau<2
$$

and the d'Alembert function must satisfy

$$
f^{\prime}(t-1) \leq 0 \quad t \in[\tau, T] \text { a.e. }
$$

Proof. By contradiction, it is shown that for $T-\tau \geq 2$ a non-grazing 1 CPP periodic motion cannot occur.

For $T-\tau=2$, the active contact duration equals the period of $u_{x}(1, t)$, which is 2-periodic through (32). In future time $t>T, u_{x}(1, t)$ can therefore be described using active contact conditions exclusively. The same applies for $T-\tau>2$. Since uniqueness is guaranteed, any motion with $T-\tau \geq 2$ remains in active contact motion for any $t>T$ and therefore never switches to inactive contact (such motion, by definition, would not be periodic). Thus, the bound (33) must hold to guarantee the existence of $1 \mathrm{CPP}$.

The restriction (34) is derived by plugging (31) into the active contact inequality condition (4).

\subsubsection{Periodicity and Admissibility conditions}

The periodicity of the solution is now determined by a difference equation involving the d'Alembert function.

Proposition 4. The displacement $u$ is T-periodic if its associated d'Alembert function satisfies

$$
f(s+T)=f_{0}(s)+f(T-1) \quad \forall s \in[-1,1] .
$$

Conversely, if $u(x, t)=f(t+x)-f(t-x)$ consists of a periodic motion then (35) must be true. Thus, $u(x, t)$ is periodic in time with period $T$ if and only if (35) holds true. Also, if (35) holds then $f^{\prime}$ is T-periodic. 
Proof. The proof is divided into three parts. First, it is shown that (35) can be derived from (5) and (6) (in their d'Alembert form). Second, we show Equation (35) implies that $f^{\prime}$ is $T$-periodic. Third, it is shown if $f$ abides (35) then $u(x, t)$ is $T$-periodic (in time) by virtue of uniqueness of the solution to the Signorini problem.

A periodic solution, such that (5) and (6) are valid, requires that

$$
\begin{aligned}
f(T+x)-f(T-x) & =f_{0}(x)+f_{0}(-x) & & \forall x \in[0,1] \\
f^{\prime}(T+x)-f^{\prime}(T-x) & =f_{0}^{\prime}(x)+f_{0}^{\prime}(-x) & & x \in[0,1] \text { a.e. }
\end{aligned}
$$

respectively. Differentiating Equation (36) with respect to $x$ yields:

$$
f^{\prime}(T+x)+f^{\prime}(T-x)=f_{0}^{\prime}(x)-f_{0}^{\prime}(-x) \quad x \in[0,1] \text { a.e. }
$$

In turn, adding (37) to (38) and subtracting (37) from (38) results in

$$
\begin{array}{ll}
f^{\prime}(T+x)=f_{0}^{\prime}(x) & x \in[0,1] \text { a.e. } \\
f^{\prime}(T-x)=f_{0}^{\prime}(-x) & x \in[0,1] \text { a.e. }
\end{array}
$$

respectively. Next, $s=x$ and $s=-x$ are substituted into (39) and (40), respectively

$$
\begin{array}{ll}
f^{\prime}(T+s)=f_{0}^{\prime}(s) & s \in[0,1] \text { a.e. } \\
f^{\prime}(T+s)=f_{0}^{\prime}(s) & s \in[-1,0] \text { a.e. }
\end{array}
$$

which can then be assembled as

$$
f^{\prime}(T+s)=f_{0}^{\prime}(s) \quad s \in[-1,1] \text { a.e. }
$$

Integrating (43) results in (35). Thus, we have shown that if (5) and (6) are valid, Equation (35) is true.

Next, $f^{\prime}$ abiding (43) (being the derivative (35)) is periodic. To prove this, we show that $f^{\prime}(s+T)=$ $f^{\prime}(s)$ for $s \in[-1, T+1]$ a.e. by assuming that $f^{\prime}(s+T)$ and $f^{\prime}(s)$ are defined by the same NDDEs and initial conditions (formulated as problem I and II).

Problem 1 The determination of $f^{\prime}(s)$ for $s \in[1, T+1]$ can be obtained from an NDDE problem formed by the inactive boundary conditions (12) and the derivative of the active contact boundary conditions (13)

\section{NDDE I}

$$
f^{\prime}(s)= \begin{cases}-f^{\prime}(s-2) & s \in[1, \tau+1] \text { a.e. } \\ f^{\prime}(s-2) & s \in[\tau+1, T+1] \text { a.e. }\end{cases}
$$

\section{Initial conditions I}

$$
f^{\prime}(s)=f_{0}^{\prime}(s) \quad s \in[-1,1] \text { a.e. }
$$

Problem II Similarly, for $s \geq T+1$, it is expected that the same boundary conditions apply. Namely, determining $f^{\prime}(s)$ for $s \in[T+1,2 T+1]$ requires solving

\section{NDDE II}

$$
f^{\prime}(s)= \begin{cases}-f^{\prime}(s-2) & s \in[1+T, \tau+T+1] \text { a.e. } \\ f^{\prime}(s-2) & s \in[\tau+T+1,2 T+1] \text { a.e. }\end{cases}
$$

where the initial conditions are assumed to be known from Equation (43)

\section{Initial conditions II}

$$
f^{\prime}(s)=f_{0}^{\prime}(s-T) \quad s \in[T-1, T+1] \text { a.e. }
$$


Note that both initial conditions I and II are given by the same function $f_{0}^{\prime}(s)$ for $s \in[-1,1]$ a.e. Similarly, NDDEs I and II are equivalent up to a phase difference T. As shown in sections 3.3.1 and 3.3.2, the solution to NDDE I is unique with respect to initial conditions (given $f_{0}(-1)=0$ as in (9)). Similarly, using the method of steps, one can show that NDDE II produces a unique solution with respect to initial conditions (given $f_{0}(-1)=0$ ). Therefore, the solutions $f^{\prime}(s)$ for $s \in[-1, T+1]$ and $f^{\prime}(s)$ for $s \in[T-1,2 T+1]$, must be equal, that is $f^{\prime}(T+s)=f^{\prime}(s), s \in$ $[-1, T+1]$ a.e. and, by induction, we can show the relationship holds for the extensions of $f^{\prime}$ in $s>2 T+1: f^{\prime}(n T+s)=f^{\prime}((n-1) T+s), s \in[-1, T+1]$ a.e., $n=2,3, \ldots, \infty$ which also means that $f^{\prime}$ is periodic: $f^{\prime}(T+s)=f^{\prime}(s), s \in[-1, \infty)$ a.e.

Finally, statement (35) shows that the state of the bar at the end of the period equals the state of the bar at the beginning of the period since $u(x, T)=f(T+x)-f(T-x)=f(x)-f(-x)=u(x, 0)$, $\forall x \in[0,1]$ and $u_{t}(x, T)=f^{\prime}(T+x)-f^{\prime}(T-x)=f^{\prime}(x)-f^{\prime}(-x)=u_{t}(x, 0), x \in[0,1]$ a.e.. Through uniqueness [15], condition (35) implies a $T$-periodic motion of the bar in dimensionless time.

\subsubsection{Summary of necessary conditions for periodic solutions}

Via the d'Alembert travelling wave solutions, the conditions for finding a periodic solution consist of functional equations and inequalities summarized below.

Conditions for Periodic Solutions of the Bar in Unilateral Contact (CPS) For $d>0, a$ $1 C P P$ motion subject to (1)-(6) and described by $u(x, t)=f(t+x)-f(t-x)$ requires finding $\tau<4$, $T<\tau+2$ and $f_{0}(s)$ continuous and piecewise $\mathcal{C}^{1}$ on $[-1,1]$ such that (equation tags are recalled)

$$
\begin{aligned}
& f_{0}(-1)=0 \\
& f_{0}(1)=d
\end{aligned}
$$

No penetration conditions for $t \in[0, \tau](u(1, t)<d)$ :

$$
\begin{array}{ll}
f_{0}(s) \geq 0 & \forall s \in[-1, \min (1, \tau-1)] \\
f_{0}(s) \leq d & \forall s \in[-1, \tau-3] \text { if } 2<\tau<4
\end{array}
$$

Positive incoming velocity and contact at $t=\tau\left(u(1, \tau)=g, u_{t}\left(1, \tau^{-}\right)>0\right)$ :

$$
\begin{aligned}
& f(\tau+1)-f(\tau-1)=d \\
& f^{\prime}\left(\tau^{-}+1\right)-f^{\prime}\left(\tau^{-}-1\right)>0
\end{aligned}
$$

Repulsion by obstacle for $t \in[\tau, T]\left(u_{x}(1, t)<0\right)$ :

$$
f^{\prime}(s) \leq 0 \quad s \in[\tau-1, T-1] \text { a.e. }
$$

\section{Periodicity condition:}

$$
f(s+T)-f(T-1)-f_{0}(s)=0 \quad \forall s \in[-1,1]
$$

where $f$ is defined by combining (17) and (31) as

$$
\tau \leq 2: \quad f(s)= \begin{cases}f_{0}(s) & s \in[-1,1] \\ d-f_{0}(s-2) & s \in[1, \tau+1] \\ d+f(s-2) & s \in[\tau+1, T+1]\end{cases}
$$

or

$$
2 \leq \tau<4: \quad f(s)= \begin{cases}f_{0}(s) & s \in[-1,1] \\ d-f_{0}(s-2) & s \in[1,3] \\ f_{0}(s-4) & s \in[3, \tau+1] \\ d+f(s-2) & s \in[\tau+1, T+1]\end{cases}
$$

While a complete closed-form solution to the CPS could not be found, it will be shown that the CPS can be solved for some cases. 


\section{Non-smooth modal analysis}

\subsection{Known properties of the non-smooth modal space}

Previous NSA on the cantilever bar have led to several important findings [20,22,23]:

F.1 Non-smooth modes parameterized by the period must be piecewise-linear and are already known to exist for $T \in(3,4)$ and $\tau \in(2,4)[22]$, see Figure 6 . The corresponding initial conditions are

$$
u_{0}(x)=\frac{d x}{3-T}, \quad v_{0}(x)=0 \quad \forall x \in[0,1]
$$

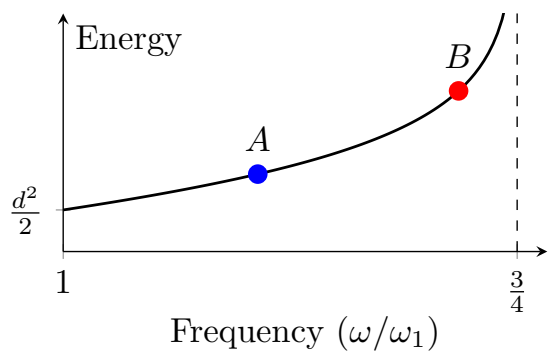

(a) Frequency energy plot. ${ }^{1}$

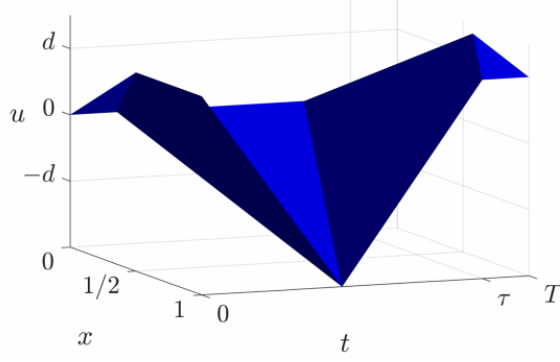

(b) Motion at point $A$.

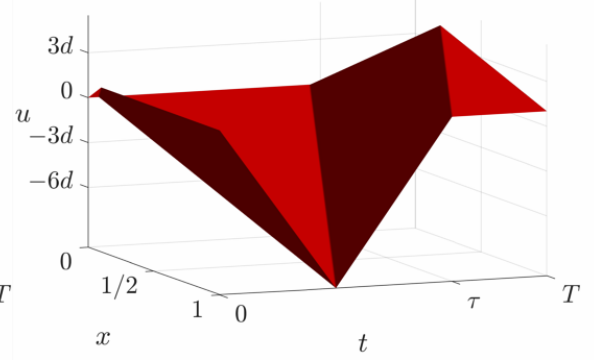

(c) Motion at point $B$.

Figure 6: Nonsmooth Mode of vibration discussed in F.1. In plot (a): $\omega=2 \pi / T$ and $\omega_{1}=2 \pi / 4$.

F.2 The NSA of the the system of interest was performed numerically in [23] through the wave finiteelement method (WFEM). From this numerical investigation, it was shown that the low-energy backbone curve in Figure 7 is continuous and can be parameterized by $T$. However, from the clouds of points, each of which represents a periodic solution, other continua of periodic solutions (e.g. internal resonances) could not be clearly established.

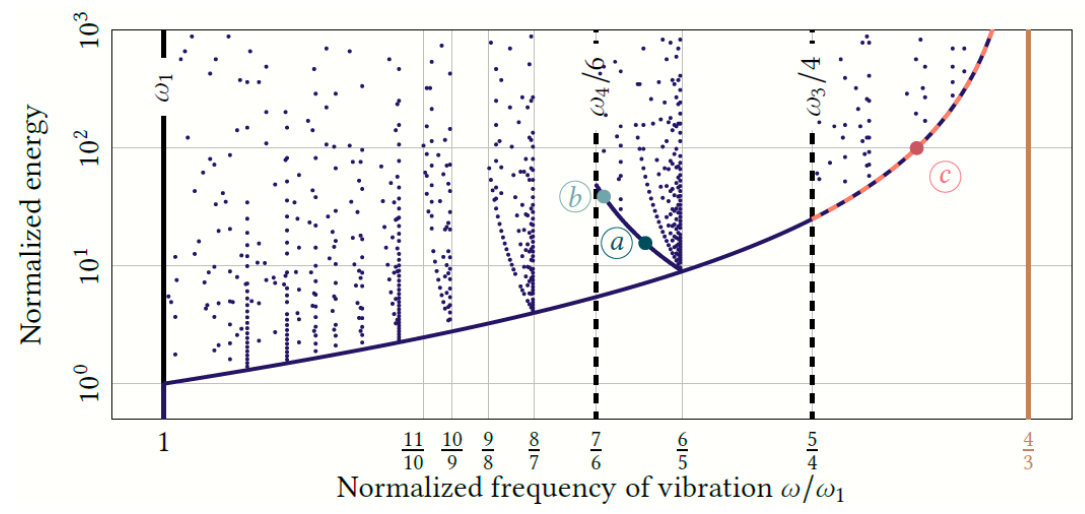

Figure 7: Energy-frequency plot corresponding to piecewise-linear mode. $\omega=2 \pi / T$ and $\omega_{1}=2 \pi / 4$. Source: [22].

F.3 In [20], vertical backbone curves of piecewise-linear modes were shown to exist for $T \in \mathbb{Q}$, as depicted in Figure 8. We note here that figures 7 and 8 seem to disagree as both approaches were not able to reveal the full modal space in the considered frequency range. This task remains unsolvable in this paper as well.

\footnotetext{
${ }^{1} \mathrm{~A}$ factor of two correction to the energy from the published article. This is explained at the footnote before equation (97). This correction has been applied to all figures and quantities affected by this.
} 


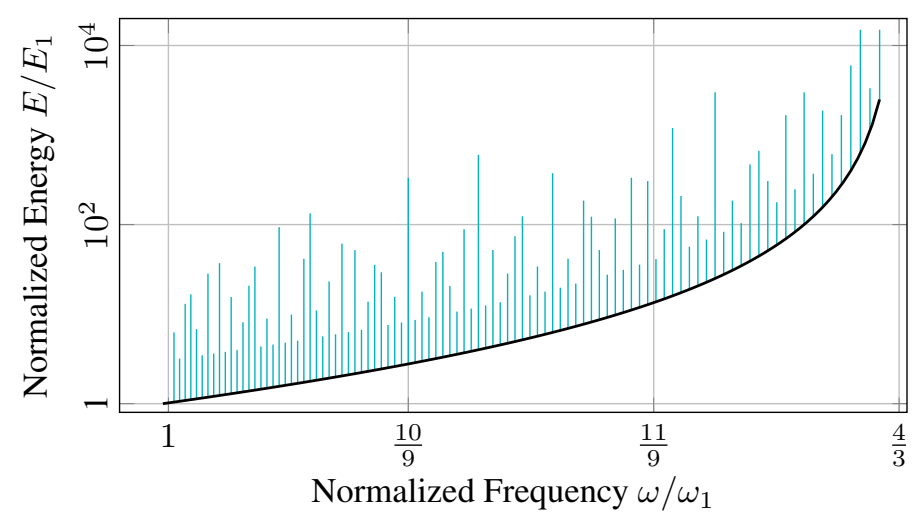

Figure 8: Families of piecewise-linear solutions with the same period of motion represented alongside the mode found in F.1. Referenced parameters: $\omega=2 \pi / T$ and $\omega_{1}=2 \pi / 4$ and $E_{1}=d^{2} / 2$. Black: piecewise linear mode in F.1. Pale-blue: piecewise-linear modes. Source: [20].

F.4 Infinitely many distinct piecewise-linear periodic solutions of the same energy, same duration of inactive contact $\tau$ and same period $T$ were shown to exist for some intervals of energy and $T \in \mathbb{Q}[20]$. Namely, for such energies and periods, there exist infinitely many initial conditions generating piecewise-linear distinct periodic solutions.

These findings, already reported in the literature, are revisited in the following sections.

\subsection{Piecewise-linear mode}

In this section, the CPS are solved to reproduce previous findings presented in F.1. Again, a known piecewise-linear mode exists for periods $T \in(3,4)$, inactive contact durations $\tau \in(2,4)$, and $2>$ $T-\tau>0$. For $\tau \in(2,4)$, the d'Alembert function is described by (49) and its last component, $f(s) \in$ $[\tau+1, T+1]$, is defined implicitly by previous components. Here, we will define the last component explicitly in $f_{0}$ using the requirement $T \in(3,4)$. We first note that for $T \in(3,4)$ and $\tau \in(2,4)$ the argument of the last component in (49), $s-2 \in[\tau+1, T+1]$, must span $s-2 \in[3,5]$ (or $s \in[1,3])$. Therefore, the last component of $f$ is determined exclusively by $f(s)$ for $s \in[1,3]$. Namely, for $\tau \in(2,4)$ and $T \in(3,4)$, we obtain

$$
f(s)=\left\{\begin{array}{ll}
f_{0}(s) & s \in[-1,1] \\
d-f_{0}(s-2) & s \in[1,3] \\
f_{0}(s-4) & s \in[3, \tau+1] \\
d+f(s-2) & s \in[\tau+1, T+1]
\end{array}= \begin{cases}f_{0}(s) & s \in[-1,1] \\
d-f_{0}(s-2) & s \in[1,3] \\
f_{0}(s-4) & s \in[3, \tau+1] \\
2 d-f_{0}(s-4) & s \in[\tau+1, T+1]\end{cases}\right.
$$

without loss of generality. Before plugging Expression (51) into the CPS, we use the knowledge that $f$ is continuous piecewise linear, see F.1, to simplify the application of the CPS. Since $f$ is piecewise linear, it is trivial to assume that $f_{0}$ is piecewise-linear as well. In order to find the loci of non-smoothness in $f_{0}$, we note that the CPS conditions (23) and (34) apply for two adjacent non-overlapping domains: $[-1, \tau-3]$ and $[\tau-3, T-3]$. Thus, it is trivial to assume that loci of non-smoothness are $\tau-3$ and $T-3$. Accordingly, we dissect $f_{0}$ into three parts spanning $[-1, \tau-3],[\tau-3, T-3]$, and $[T-3,1]$ to obtain a piecewise-linear and continuous function of the type

$$
f_{0}(s)= \begin{cases}a_{0}+b_{0} s & s \in[-1, \tau-3] \\ a_{0}+b_{0}(\tau-3)+b_{1}(s-\tau+3) & s \in[\tau-3, T-3] \\ a_{0}+b_{0}(\tau-3)+b_{1}(T-\tau)+b_{2}(s-T+3) & s \in[T-3,1] .\end{cases}
$$


Note that other loci of non-smoothness may be possible, as shown in section 4.3. Then, the piecewiselinear $f_{0}(52)$ is plugged into the CPS conditions such that

$$
\begin{array}{ll}
\text { Eq.(9) } & \Rightarrow a_{0}=b_{0} \\
\text { Eq.(22, 23, 29) } & \Rightarrow b_{0}=d /(\tau-2) \\
\text { Eq.(14,53,55) } & \Rightarrow b_{2}=-b_{1}(T-\tau) /(4-T) \\
\text { Eq.(29, 30,34) } & \Rightarrow b_{1}>0 .
\end{array}
$$

Expressions (53)-(55) are plugged back into (52), itself inserted in (51), to form an expression of $f$ dependent on $b_{1}, \tau$ and $T$ only

$$
f(s)= \begin{cases}\frac{d}{\tau-2}(s+1) & s \in[-1, \tau-3] \\ d+b_{1}(s-\tau+3) & s \in[\tau-3, T-3] \\ d+b_{1} \frac{(T-\tau)}{4-T}(1-s) & s \in[T-3,1] \\ d-\frac{d}{\tau-2}(s-1) & s \in[1, \tau-1] \\ -b_{1}(s-\tau+1) & s \in[\tau-1, T-1] \\ -b_{1} \frac{(T-\tau)}{4-T}(3-s) & s \in[T-1,3] \\ \frac{d}{\tau-2}(s-3) & s \in[3, \tau+1] \\ d-b_{1}(s-\tau-1) & s \in[\tau+1, T+1] .\end{cases}
$$

It is noted that the first component of $f$ does not depend on $b_{1}$ since Equations (9) (which defines the reference point for $f$ ) and (29) (which establishes contact with the rigid wall at the beginning of the active contact motion) are sufficient to determine the constants $a_{0}$ and $b_{0}$.

Next, we will show that the free parameter $b_{1}$ becomes a mono-valued function of $\tau$ as soon as periodicity is enforced. For the d'Alembert function, periodicity (35) reads

$$
f_{p}(s) \equiv f(s+T)-f(T-1)=f_{0}(s) \quad \forall s \in[-1,1]
$$

where $f_{p}$ denotes the section of the d'Alembert function defining the motion at the end of the period, $f(s+T)-f(T-1)$. Based on the d'Alembert function (57), $f_{p}$ reads

$$
f_{p}(s)= \begin{cases}-b_{1} \frac{T-\tau}{4-T}(3-s-T)+b_{1}(T-\tau) & s \in[-1,3-T] \\ \frac{d}{\tau-2}(s-3+T)+b_{1}(T-\tau) & s \in[3-T, \tau-T+1] \\ d-b_{1}(s-\tau-1+T)+b_{1}(T-\tau) & s \in[\tau-T+1,1]\end{cases}
$$

and, accordingly, $f_{0}$ reads

$$
f_{0}(s)= \begin{cases}\frac{d}{\tau-2}(s+1) & s \in[-1, \tau-3] \\ d+b_{1}(s-\tau+3) & s \in[\tau-3, T-3] \\ d+b_{1} \frac{T-\tau}{4-T}(1-s) & s \in[T-3,1] .\end{cases}
$$

Naturally, the first requirement for equality of $f_{p}$ and $f_{0}$ is that their loci of non-smoothness will be congruent. Currently, the components in (59) and (60) do not span identical domains. It is chosen to establish a relationship between $\tau$ and $T$ such that the loci of non-smoothness align. Here, equality between $f_{p}$ and $f_{0}$ can be enforced as soon as the domains of definition (or support) of the last component of each function are identical, i.e. $T-3=\tau-T+1$ or

$$
2 T(\tau)=\tau+4
$$

This relationship also agrees with the piecewise-linear mode reproduced in this section [22]. Inserting (61) into (59) and (60) yields:

$$
f_{p}(s)= \begin{cases}b_{1}(1+s) & s \in[-1,3-T(\tau)] \\ \frac{d}{2 T(\tau)-6}(s-3+T(\tau))+b_{1}(4-T(\tau)) & s \in[3-T(\tau), T(\tau)-3] \\ d+b_{1}(1-s) & s \in[T(\tau)-3,1]\end{cases}
$$


and

$$
f_{0}(s)= \begin{cases}\frac{d}{2 T(\tau)-6}(s+1) & s \in[-1,2 T(\tau)-7] \\ d+b_{1}(s-2 T(\tau)+7) & s \in[2 T(\tau)-7, T(\tau)-3] \\ d+b_{1}(1-s) & s \in[T(\tau)-3,1] .\end{cases}
$$

For a general solution applicable to any $\tau \in(2,4)$, the first components of $f_{0}$ and $f_{p}$ overlap. For $f_{0}=f_{p}$, the first components must have the same slope, that is

$$
b_{1}(\tau)=\frac{d}{2 T(\tau)-6}
$$

As a consequence, the first and second components in $f_{0}$ and in $f_{p}$ may be merged to form

$$
f_{0}(s)=f_{p}(s)=\frac{d}{2 T(\tau)-6} \begin{cases}s+1 & s \in[-1, T(\tau)-3] \\ 2 T(\tau)-5-s & s \in[T(\tau)-3,1]\end{cases}
$$

and the periodicity condition (35) is satisfied for arbitrary $\tau \in(2,4)$ and $T \in(3,4)$. Thus, any initial condition (65), for the aforementioned $T$ and $\tau$, generates a periodic motion. This piecewise-linear mode is now properly defined.

NSM1: Piecewise-Linear Mode of the Cantilever Bar in Unilateral Contact Given $d>0$, $u(x, t)=f(t+x)-f(t-x)$ with $f$ such that

$$
f(s)=\frac{d}{2 T-6} \begin{cases}s+1 & s \in[-1, T-3] \\ 2 T-5-s & s \in[T-3, T-1] \\ s-3 & s \in[T-1,2 T-3] \\ 4 T-9-s & s \in[2 T-3, T+1]\end{cases}
$$

represents a non-smooth nonlinear mode for all $T \in[3,4]^{2}$.

To show NSM1 is equivalent to the piecewise-linear mode introduced in F.1, it is noted that NSM1 at $t=T-2$ generates the displacement and velocity fields

$$
\begin{aligned}
u(x, T-2) & =f(T-2+x)-f(T-2-x) \\
& =\frac{d}{2 T-6}(T-3-x)-\frac{d}{2 T-6}(2 T-3+x)=-\frac{d x}{T-3}, \quad \forall x \in[0,1] \\
u_{t}(x, T-2) & =f^{\prime}(T-2+x)-f^{\prime}(T-2-x) \\
& =-\frac{d}{2 T-6}+\frac{d}{2 T-6}=0, \quad \forall x \in[0,1]
\end{aligned}
$$

which agree with (50). Since the solution is unique with respect to initial condition [15], NSM1 is indeed equivalent to the piecewise-linear mode presented in F.1. Furthermore, we note that NSM1 exists for both rational and irrational periods $T \in[3,4]$. This fact has been also established in [1] where it is stated that a continuum of periodic solutions continuously spanning different periods must consist of piecewise-linear displacements. However, it has been speculated in [1] that for the case of rational $T$, families of periodic solutions that are not piecewise-linear may exist. Here, using the d'Alembert formulation and the CPS, we have found some of such other families of periodic solutions.

\footnotetext{
${ }^{2}$ Note that we have removed any reference to $\tau$ as it is not critical for illustrating the mode NSM1.
} 


\subsection{Piecewise-smooth mode(s) of the same period}

For $T \in \mathbb{Q}$, previous works have only reported piecewise-linear modes. Here, we show that solutions that are piecewise-smooth exist as well. This section details a methodology for determining analytically these piecewise-smooth modes. An illustrative example is provided.

Assume that both $T$ and $\tau$ belong to $\mathbb{Q}(\tau \in \mathbb{Q}$ is also a consequence of the work in [20]) such that $T$ and $\tau$ can be described by $n, p<n$, and $m$ belonging to $\mathbb{N}$, namely,

$$
T=n / m, \quad \tau=p / m .
$$

To accommodate for the CPS conditions spanning non-uniform domains, such that no penetration conditions (22)-(23) and negative repulsion force condition (34), it is useful to dissect $f_{0}$ into $2 m$ equal components, each consisting of a smooth function $a_{i}(s)$

$$
f_{0}(s)= \begin{cases}a_{0}(s+1) & s \in[-1,-1+1 / m] \\ a_{1}(s+1-1 / m) & s \in[-1+1 / m,-1+2 / m] \\ \vdots & s \in[-1+i / m,-1+(i+1) / m] \\ a_{i}(s+1-i / m) & \\ \vdots & s \in[1-1 / m, 1]\end{cases}
$$

where the functions $a_{i}$ have the following features:

$$
\begin{aligned}
& a_{i}:[0,1 / m] \mapsto[0, d], \quad i=0,1, \ldots, p-2 m-1 \\
& a_{i}:[0,1 / m] \mapsto[0, \infty) \quad i=p-2 m, p-2 m+1, \ldots, 2 m-1 .
\end{aligned}
$$

These functions can be nonlinear in $s$ in contrast with (52) where $a_{0}$ stands for a constant. The condition $a_{i}(s) \geq 0$ is a consequence of (22). In this paper, only 1CPP with $\tau>2$ are considered so $a_{i}(s) \leq d$ from (23). Moreover, to preserve the continuity of $f_{0}$, the following must hold: $a_{i-1}(1 / m)=$ $a_{i}(0)$ for all $i=1,2, \ldots, 2 m-2$. This dissection of $f_{0}$ allows then for easy depiction of the solution space of the CPS for $T \in \mathbb{Q}$.

As an example, a solution for $n=10, p=8$ and $m=3$, i.e. inactive motion duration $\tau=8 / 3$ and period $T=10 / 3$, is derived in the remainder of this section. For other choice of parameters $p, n$ and $m$ this method may fail as further discussed in section 4.4. Inserting the above $\tau$ and $T$ together with (72) into (49) leads to

$$
f(s)= \begin{cases}a_{0}(s+1) & s \in[-1,-2 / 3] \\ a_{1}(s+2 / 3) & s \in[-2 / 3,-1 / 3] \\ a_{2}(s+1 / 3) & s \in[-1 / 3,0] \\ a_{3}(s) & s \in[0,1 / 3] \\ a_{4}(s-1 / 3) & s \in[1 / 3,2 / 3] \\ a_{5}(s-2 / 3) & s \in[2 / 3,1] \\ d-a_{0}(s-1) & s \in[1,4 / 3] \\ d-a_{1}(s-4 / 3) & s \in[4 / 3,5 / 3] \\ d-a_{2}(s-5 / 3) & s \in[5 / 3,2] \\ d-a_{3}(s-2) & s \in[2,7 / 3] \\ d-a_{4}(s-7 / 3) & s \in[7 / 3,8 / 3] \\ d-a_{5}(s-8 / 3) & s \in[8 / 3,3] \\ a_{0}(s-3) & s \in[3,10 / 3] \\ a_{1}(s-10 / 3) & s \in[10 / 3,11 / 3] \\ 2 d-a_{2}(s-11 / 3) & s \in[11 / 3,4] \\ 2 d-a_{3}(s-4) & s \in[4,13 / 3]\end{cases}
$$


Note that the most intricate functional equation in the CPS involves the periodicity condition (35)

$$
f_{p}(s) \equiv f(s+T)-f(-s+T)=f_{0}(s)
$$

where $f_{p}$ has been added to denote the state of the bar at the end of contact. Imposing the periodicity condition (76) on the form of $f$ in (75) leads to

$$
f_{p}(s)= \begin{cases}-a_{4}(s+1)+a_{4}(0) & s \in[-1,-2 / 3] \\ -a_{5}(s+2 / 3)+a_{4}(0) & s \in[-2 / 3,-1 / 3] \\ a_{0}(s+1 / 3)+a_{4}(0)-d & s \in[-1 / 3,0] \\ a_{1}(s)+a_{4}(0)-d & s \in[0,1 / 3] \\ -a_{2}(s-1 / 3)+a_{4}(0)+d & s \in[1 / 3,2 / 3] \\ -a_{3}(s-2 / 3)+a_{4}(0)+d & s \in[2 / 3,1]\end{cases}
$$

and

$$
f_{0}(s)= \begin{cases}a_{0}(s+1) & s \in[-1,-2 / 3] \\ a_{1}(s+2 / 3) & s \in[-2 / 3,-1 / 3] \\ a_{2}(s+1 / 3) & s \in[-1 / 3,0] \\ a_{3}(s) & s \in[0,1 / 3] \\ a_{4}(s-1 / 3) & s \in[1 / 3,2 / 3] \\ a_{5}(s-2 / 3) & s \in[2 / 3,1] .\end{cases}
$$

The solution to (76) can be easily extracted by equating each of the components in $f_{0}$ to their corresponding components in $f_{p}$ :

$$
\begin{array}{ll}
a_{0}(s)=a_{4}(0)-a_{4}(s)=a_{2}(s)-d=a_{0}(s)+a_{4}(0)-2 d, & \forall s \in[0, L / 3] \\
a_{1}(s)=a_{4}(0)-a_{5}(s)=a_{3}(s)-d=a_{1}(s)+a_{4}(0)-2 d, & \forall s \in[0, L / 3] .
\end{array}
$$

For the equalities to hold true, $a_{4}(0)=2 d$ must hold. The dissection of $f_{0}$ and periodicity condition are visualized in Figure 9. Thus, applying all other conditions in the CPS (besides the already solved periodicity condition), a non-smooth mode is obtained as described below.

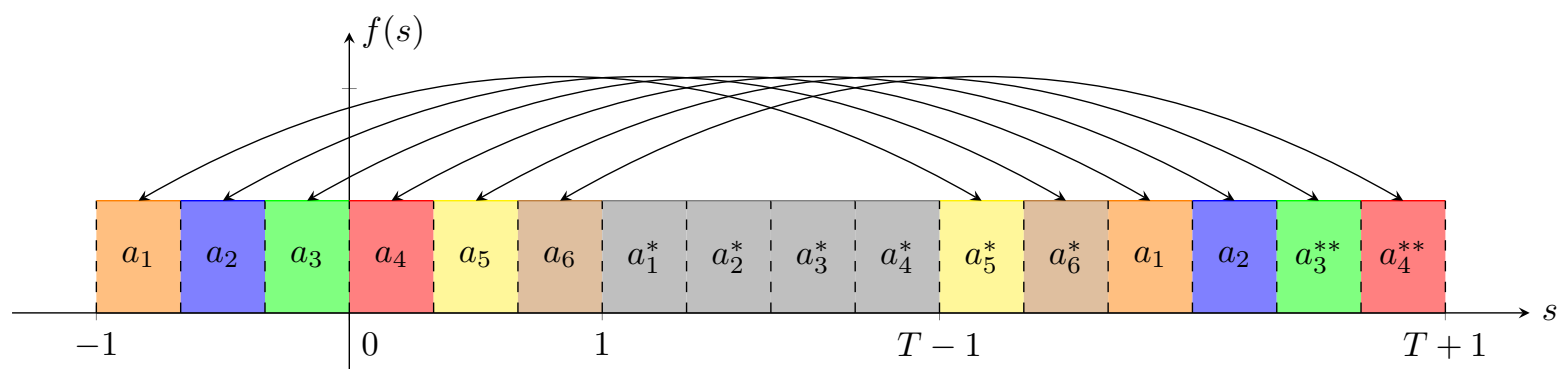

Figure 9: Method for finding piecewise-smooth solutions. $f_{0}$ is a piecewise-smooth continuous function where all smooth components span domains of equal length $1 / m$. As expressed in (48) or (49), the extension of the d'Alembert function is done via affine transformations. For the specific $f$ in (75): $a_{i}^{*}(s)=d-a_{i}(s), a_{i}^{* *}(s)=2 d-a_{i}(s)$. To satisfy periodicity (35), the non-gray components are equated as done in (79) and (80).

NSM2: Piecewise-Smooth Mode of the Cantilever Bar in Unilateral Contact For an inactive contact duration $\tau=8 / 3$ and period $T=10 / 3$, the arbitrary piecewise-smooth functions $a_{0}(s)$ and $a_{1}(s)$ in the domain $s \in[0,1 / 3]$, with the constraints

Continuity of $f_{0}: a_{0}(1 / 3)=a_{1}(0)$

$$
\begin{aligned}
E q .(9,29): & a_{0}(0)=0 \\
E q .(14): & a_{1}(1 / 3)=d
\end{aligned}
$$


and, recalling the remaining CPS conditions,

$$
\begin{array}{lll}
E q \cdot(30) & \Rightarrow \quad a_{0}^{\prime}(0)>0 & \\
E q \cdot(22,23) & \Rightarrow 0 \leq a_{0}(s) \leq d, & \forall s \in[0,1 / 3] \\
E q .(22,23) & \Rightarrow 0 \leq a_{1}(s) \leq d, & \forall s \in[0,1 / 3] \\
E q .(34) & \Rightarrow a_{0}^{\prime}(s) \geq 0, & \forall s \in[0,1 / 3] \\
E q .(34) & \Rightarrow a_{1}^{\prime}(s) \geq 0, & \forall s \in[0,1 / 3]
\end{array}
$$

define a non-smooth mode with $u(x, t)=f(t+x)-f(t-x)$ where $f$ is defined in (49) with initial condition

$$
f_{0}(s)= \begin{cases}a_{0}(s+1) & s \in[-1,-2 / 3] \\ a_{1}(s+2 / 3) & s \in[-2 / 3,-1 / 3] \\ d+a_{0}(s+1 / 3) & s \in[-1 / 3,0] \\ d+a_{1}(s) & s \in[0,1 / 3] \\ 2 d-a_{0}(s-1 / 3) & s \in[1 / 3,2 / 3] \\ 2 d-a_{1}(s) & s \in[2 / 3,1] .\end{cases}
$$

NSM2, as shown in Figures 10 and 11, consists of periodic motions existing exclusively for $T=10 / 3$. From conditions (81)-(88) on functions $a_{0}$ and $a_{1}$, we understand that NSM2 exists in a convex (because of inequalities) subspace of $\left(C^{1}[0,1 / 3]\right)^{2}$. In turn, using the procedure presented in this section with different $m, n$ and $p$, similar mode can be derived for any period of the type $T \in \mathbb{Q}$ and $\tau \in \mathbb{Q}$. However, for some values of $m, n$ and $p$, the proposed methodology could not generate periodic solutions. Also, choosing affine functions $a_{0}$ and $a_{1}$ replicates the results exposed in [20].

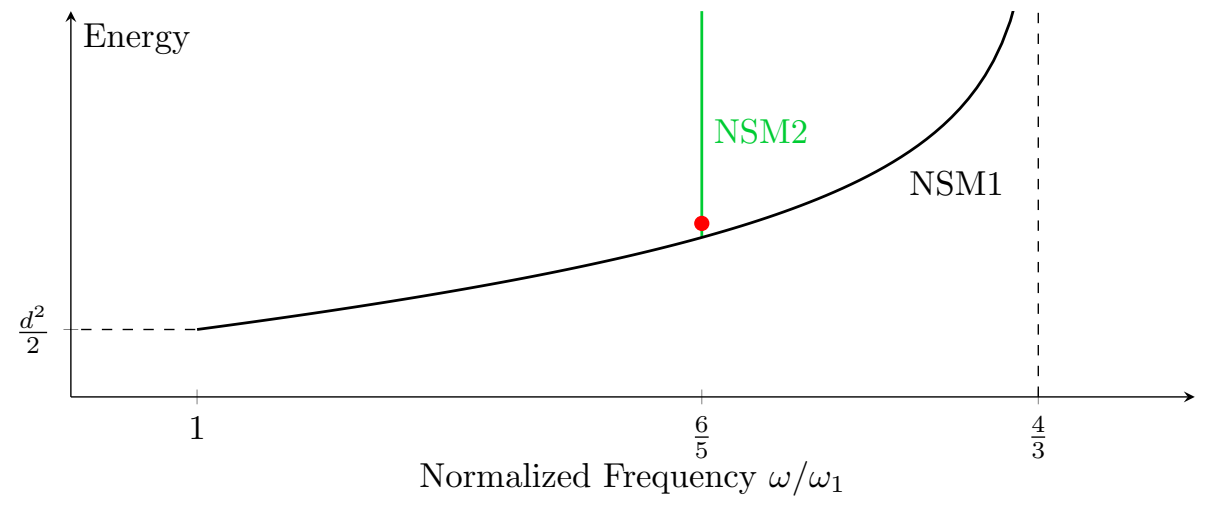

Figure 10: Piecewise-smooth mode NSM2 in comparison to NSM1. $\omega=2 \pi / T$ and $\omega_{1}=2 \pi / 4$. The point in red indicates a mode of solutions of the same energy, described in the proof of Theorem 7 .

\section{Example of NSM2 solutions}

The functions $a_{0}$ and $a_{1}$ involved in the definition of NSM2 exist in a convex subspace of $\left(C^{1}[0,1 / 3]\right)^{2}$. It is of interest to give an example of a functional space with varying $a_{0}$ and constant $a_{1}$ answering all NSM2 equalities and inequalities (81) to (88). To this end, we choose an arbitrary real function $R_{0}(s)$ such that

$$
a_{0}(s)=d \int_{0}^{s} R_{0}^{2}(\mu) \mathrm{d} \mu, \quad s \in[0,1 / 3] \quad \text { and } \quad \int_{0}^{1 / 3} R_{0}^{2}(\mu) \mathrm{d} \mu=1
$$

together with

$$
a_{1}(s)=d \text {. }
$$




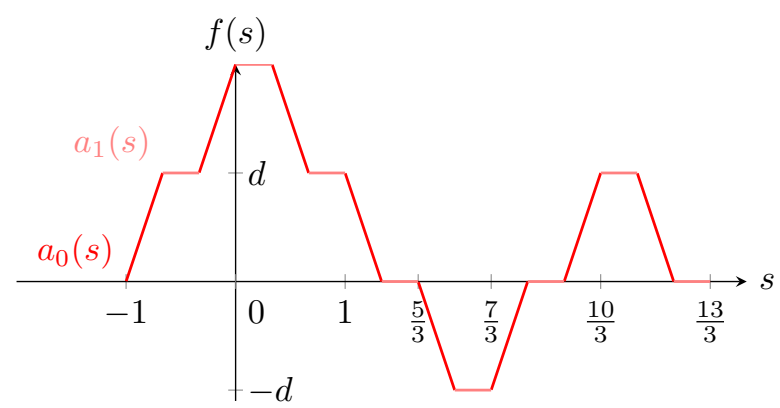

(a) D'Alembert function for $a_{0}(s)=3 d s$ (or $R_{0}=1$ in reference to section 4.3) and $a_{1}(s)=d$.

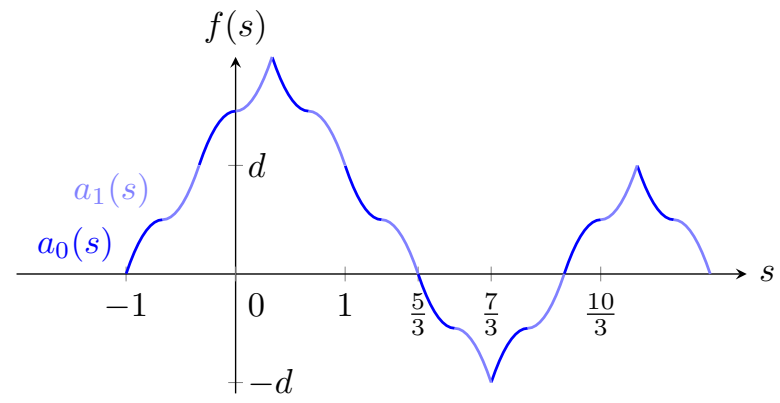

(c) d'Alembert function for $a_{0}(s)=-4.5 d\left((s-1 / 3)^{2}-\right.$ $\left.(1 / 3)^{2}\right)$ and $a_{1}(s)=4.5 d s^{2}+0.5 d$.

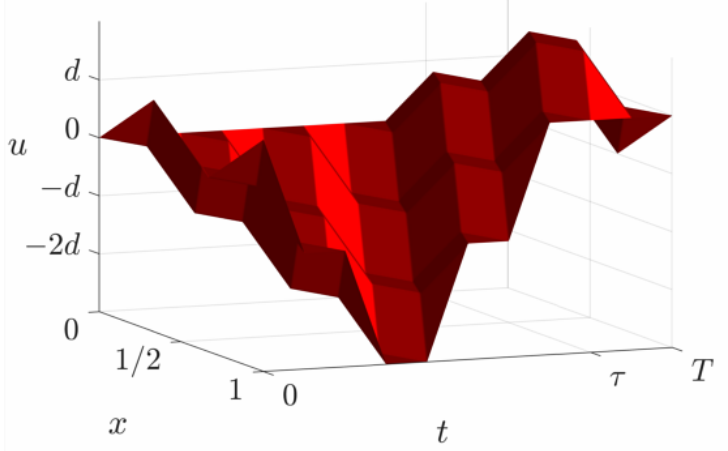

(b) Displacement.

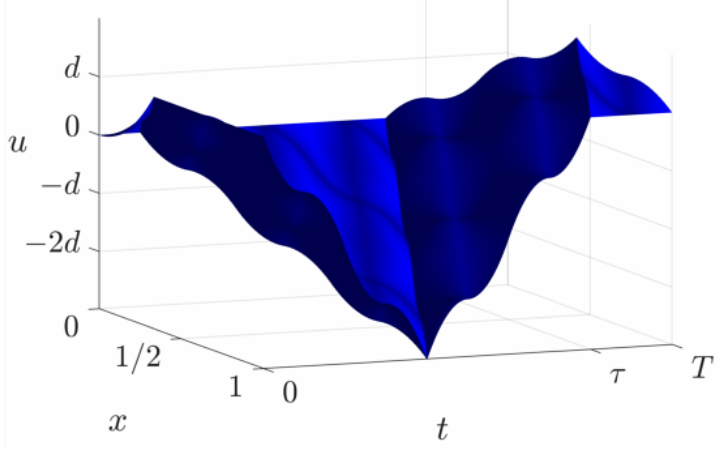

(d) Displacement.

Figure 11: Periodic motions belonging to NSM2.

Note that for any function $R_{0}$, Equations (90) and (91) satisfy NSM2 conditions (81)-(83) and (85)(88). The remaining condition (84) is satisfied by restricting $R_{0}$ as follows:

$$
R_{0}(0) \neq 0 \text {. }
$$

Thus, any integrable function $R_{0}$, such that (90) to (92) are satisfied, generates a periodic solution of the type NSM2 (including piecewise-smooth). Such a motion is illustrated in Figure 11(a-b).

\subsection{Applications to non-smooth modal analysis}

The use of d'Alembert function has proven useful in deriving novel periodic solutions, see NSM2. While non-smooth modes of the bar in unilateral contact were considered to consist mainly of piecewise-linear functions (F.1 to F.4), it has been shown that other piecewise-smooth solutions may exist as well.

Theorem 5. There exist piecewise-smooth modes of the cantilever bar in unilateral contact which are not necessarily piecewise-linear.

Proof. An example of such piecewise smooth mode is NSM2 in section 4.3. Using the same methodology as presented in 4.3 , with different values $p, n$ and $m$, it is possible to find other modes of the same type.

Conjecture 6. For all $T \in \mathbb{Q}$ where a periodic motion of the cantilever bar in unilateral contact exists, there exists a piecewise-smooth mode of the type discussed in Theorem 5.

As mentioned previously, using the methodology in section 4.3, we have found solutions for other $T \in \mathbb{Q}$. In fact, we can confidently conjecture that there exist piecewise-smooth modes for a dense group of $T \in \mathbb{Q}$. Conjecture 6 is also supported analytically in $[1$, p.7] where it is stated that any mode that is not piecewise-linear would exist only for $T \in \mathbb{Q}$.

At last, the detected modes discussed in conjecture 6 exhibit a peculiar property: infinitely many periodic solutions for the same energy and same period, as suggested in F.4 and proved below. 
Theorem 7. For the cantilever bar in unilateral contact, there exist continua of piecewise-smooth periodic solutions of the same energy and frequency.

Proof. Consider the dimensionless energy of the cantilever bar:

$$
2 E(t)=\int_{0}^{1} u_{x}^{2}(x, t)+u_{t}^{2}(x, t) \mathrm{d} x
$$

Plugging the d'Alembert expression of the stress and velocity (8) gives:

$$
\begin{aligned}
2 E(t) & =\int_{0}^{1}\left(f^{\prime}(t+x)+f^{\prime}(t-x)\right)^{2}+\left(f^{\prime}(t+x)-f^{\prime}(t-x)\right)^{2} \mathrm{~d} x \\
& =\int_{0}^{1} f^{\prime 2}(t+x) \mathrm{d} x+\int_{0}^{1} f^{\prime 2}(t-x) \mathrm{d} x
\end{aligned}
$$

The second term can be put then in terms of $f^{\prime}(t+x)$ via a basic change of variable

$$
2 E(t)=\int_{0}^{1} f^{\prime 2}(t+x) \mathrm{d} x-\int_{0}^{-1} f^{\prime 2}(t+x) \mathrm{d} x=\int_{-1}^{1} f^{\prime 2}(t+x) \mathrm{d} x=\int_{t-1}^{t+1} f^{\prime 2}(s) \mathrm{d} s .
$$

Also, note that the energy under unilateral contact is preserved $[3,19,23]$ and can be represented in terms of initial conditions, in the d'Alembert form $f_{0}$, exclusively ${ }^{3}$

$$
E(t)=E(0)=\int_{-1}^{1} f_{0}^{\prime 2}(s) \mathrm{d} s \equiv E
$$

Plugging $f_{0}$ from NSM2 (89) leads to (the simplification procedure has been omitted for sake of (conciseness) $)^{4}$

$$
E=3 \int_{0}^{1 / 3} a_{0}^{\prime 2}(s) \mathrm{d} s+3 \int_{0}^{1 / 3} a_{1}^{\prime 2}(s) \mathrm{d} s .
$$

Any functions $a_{1}$ and $a_{0}$ satisfying (98) generate a periodic solution with $T=10 / 3, \tau=8 / 3$ and prescribed energy $E$. For example, a linear function $a_{0}$ and cubic function $a_{1}$ belonging to NSM2 can be denoted as follows (derivations were omitted to facilitate reading)

$$
\begin{aligned}
& a_{0}(s)=b_{1} s \\
& a_{1}(s)=27\left(d-\frac{b_{1}}{3}\right) \frac{s^{3}+b_{2} s}{1+9 b_{2}}+\frac{b_{1}}{3}
\end{aligned}
$$

where, to satisfy (81)-(88), $b_{1}$ and $b_{2}$ must abide

$$
\begin{aligned}
0<b_{1} & \leq 3 d, \\
b_{2} & >0 .
\end{aligned}
$$

To obtain the energy of this NSM2 motion, (99) and (100) are plugged into (98):

$$
E\left(b_{1}, b_{2}\right)=\frac{9\left(45 b_{2}^{2}+10 b_{2}+1\right)\left(b_{1}-3 d\right)^{2}}{5\left(9 b_{2}+1\right)^{2}}+b_{1}^{2}
$$

For several energy values, $E$, there exist infinitely many values $b_{1}$ and $b_{2}$ (i.e. infinitely many NSM2 solutions) generating motions of the same energy. For example, $E\left(b_{1}, b_{2}\right)=9 d^{2}$ is satisfied for any

$$
b_{1}=\frac{6 d}{7+90 b_{2}+405 b_{2}^{2}}
$$

and $0<b_{2}<\infty$ satisfies (101) and (102). Thus, $b_{2} \in(0, \infty]$ with (104), (99) and (100) represents a family of periodic solution with the same energy and period in NSM2. Several solutions from this piecewise-smooth mode are depicted in Figure 12.

\footnotetext{
${ }^{3}$ This is the correct formula for the energy in comparison to the same formula in the published article

${ }^{4} \mathrm{~A}$ factor of three has been omitted in the published article. All other mentions of energy depending on this formulation have been corrected as well.
} 


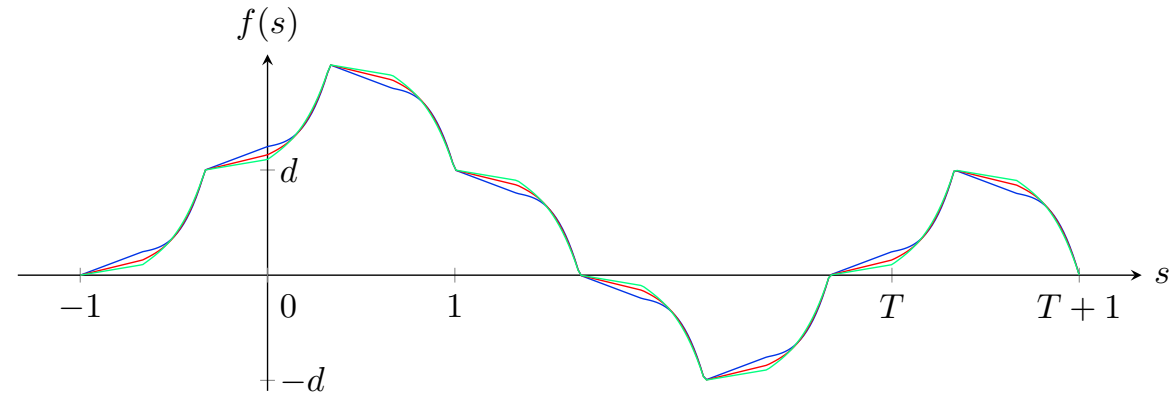

(a) D'Alembert functions for selected $b_{2}$ values. $b_{2}=d / 5[-] b_{2}=3 d / 5[-]$, and $b_{2}=d[-]$.

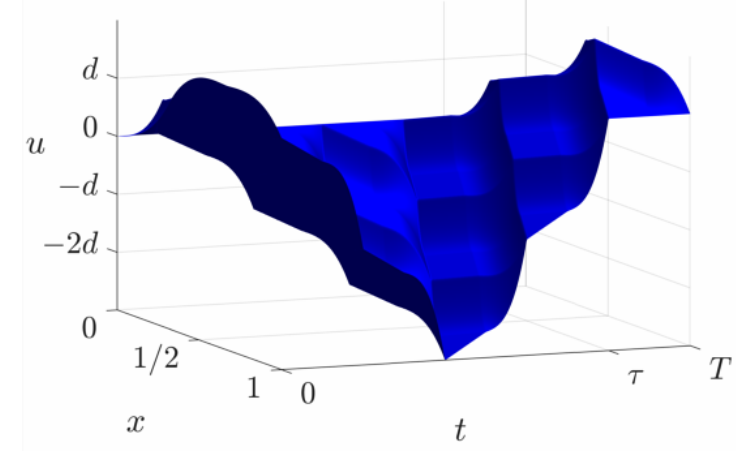

(b) Displacement field, $b_{2}=d / 5$.

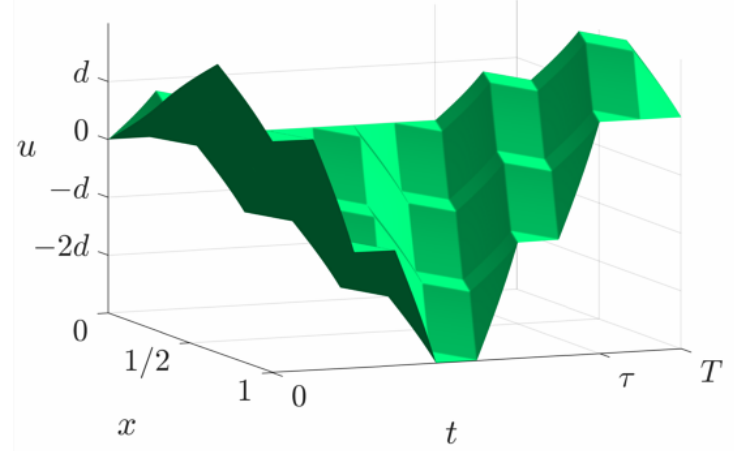

(c) Displacement field, $b_{2}=d$.

Figure 12: Selected solutions from the continuum of solutions described by (99), (100) and (104) defined by the $b_{2}$ values. All depicted solutions are motions of energy $E=9 d^{2}$ and period $T=10 / 3$.

Similarly to Conjecture 6 , for $\tau, T \in \mathbb{Q}$ where periodic motions can be found, there also exist energy-frequency (or period) pairs consisting of piecewise-smooth modes of the type mentioned in Theorem 7. This mode is also depicted as a red point in Figure 10.

The theorems and conjectures presented in this section are used to derive conclusions about NSA via numerical methods:

C.1 The aforementioned "isolated" solutions presented in F.2 most likely belong to the non-smooth modes of the type discussed in Theorem 5. This conclusion is induced by the fact that the numerical method used to obtain the isolated solutions in F.2 produces Signorini solutions for rational periods and inactive contact durations $T, \tau \in \mathbb{Q}$ similar to the piecewise-smooth modes discussed in Theorem 5 .

C.2 The non-smooth modes in the present work can be parameterized in functional spaces describing different possible initial conditions $f_{0}$ generating motions that are not necessarily piecewise-linear yet can be of the same energy and and of the same period. Here, conjecture 6 implies that these modes exist for a dense set of rational periods. This is explained by the full internal resonance condition enjoyed by the investigated one-dimensional bar. This conclusion could not be deduced by available numerical techniques [19,22].

\section{Conclusion}

The non-smooth modal analysis of a bar in unilateral contact with a rigid foundation at one boundary is explored in the present paper. Existing results already reported in the literature are extended by formulating the conditions for one-contact per period motions in terms of the d'Alembert travelling wave solution. This formulation results in a non-smooth Delay Differential Equation of the neutral type. Using the method of steps, this equation is solved and the problem of non-smooth modal analysis is transformed into a system of functional equalities and inequalities to be satisfied by the initial condition. While a complete closed-form solution cannot be exhibited, interesting solutions 
could be obtained for rational periods $T \in \mathbb{Q}$ : modes with piecewise-smooth displacement in space and time, families of modes with same period, and families of modes with the same energy and period.

The findings in this paper may also provide practical conclusions on the forced motions of the bar. Vibratory resonances induced by periodic external forcing are expected to occur at frequencies where non-smooth modes exist. Accordingly, the existence of iso-period modes for a dense group of periods $T \in \mathbb{Q}$ suggests that resonances can emerge for all periods of forcing $T \in \mathbb{Q}$. It should also be understood that the initial conditions generating admissible solutions were chosen in the space of continuous and piecewise once-differentiable functions $\mathcal{C}^{0} \cap \hat{\mathcal{C}}^{1}$ which could be extended to $\mathcal{H}^{1}$ allowing for much more exotic solutions with cusps for instance.

At last, we note that NSA via d'Alembert functions and method of steps can also be used for analysis of motions with $k>1$ active contact phases per period. Results for $k=2$ could be produced and confirm the suggested theorems and conjectures presented in this manuscript.

\section{References}

[1] C. Bertrand. Periodic solutions of a one-dimensional elastic bar subject to a unilateral constraint. Technical report, ENTPE, 2020. oai:hal-02897846 ๖.

[2] B. Brogliato. Nonsmooth Mechanics: Models, Dynamics and Control, volume 2. Springer, 1999. doi: 10.1007/978-1-4471-0557-2.

[3] D. Doyen, A. Ern, and S. Piperno. Time-integration schemes for the finite element dynamic Signorini problem. SIAM Journal on Scientific Computing, 33(1):223-249, 2011. oai:hal-00440128 ๑. doi:10. $1137 / 100791440$.

[4] T. Erneux. Applied Delay Differential Equations. Springer, 2009. doi:10.1007/978-0-387-74372-1.

[5] L.C. Evans. Partial Differential Equations. American Mathematical Society, 2010.

[6] G. James, V. Acary, and F. Pérignon. Periodic motions of coupled impact oscillators. In R.I. Leine, V. Acary, and O. Brüls, editors, Advanced Topics in Nonsmooth Dynamics: Transactions of the European Network for Nonsmooth Dynamics, pages 93-134. Springer, 2018. oai:hal-01660891 ə. doi:10.1007/ 978-3-319-75972-2_3.

[7] G. Kerschen. Definition and fundamental properties of nonlinear normal modes. In G. Kerschen, editor, Modal Analysis of Nonlinear Mechanical Systems, volume 555, pages 1-46. Springer, 2014. doi:10.1007/ 978-3-7091-1791-0_1.

[8] G. Lebeau and M. Schatzman. A wave problem in a half-space with a unilateral constraint at the boundary. Journal of Differential Equations, 53(3):309-361, 1984. oai:hal-01294216 ə. doi:10.1016/ 0022-0396 (84)90030-5.

[9] M. Legrand, S. Junca, and S. Heng. Nonsmooth modal analysis of a $N$-degree-of-freedom system undergoing a purely elastic impact law. Communications in Nonlinear Science and Numerical Simulation, 45:190-219, 2017. oai:hal-01185980 ə. doi:10.1016/j.cnsns.2016.08.022.

[10] EH. Moussi, S. Bellizzi, B. Cochelin, and I. Nistor. Nonlinear normal modes of a two degreesof-freedom piecewise linear system. Mechanical Systems and Signal Processing, 64-65:266-281, 2015. oai:hal-00783088 ə. doi:10.1016/j.ymssp.2015.03.017.

[11] M. P. Païdoussis and C. Semler. Nonlinear and chaotic oscillations of a constrained cantilevered pipe conveying fluid: A full nonlinear analysis. Nonlinear Dynamics, 4(6):655-670,1993. doi:10.1007/BF00162236.

[12] M. Peeters, R. Viguié, G. Sérandour, G. Kerschen, and J.-C. Golinval. Nonlinear normal modes, part II: Toward a practical computation using numerical continuation techniques. Mechanical Systems and Signal Processing, 23(1):195-216, 2009. oai:hal-01581480 ə. doi:10.1016/j.ymssp.2008.04.003.

[13] S. Peter, F. Schreyer, and R.I. Leine. A method for numerical and experimental nonlinear modal analysis of nonsmooth systems. Mechanical Systems and Signal Processing, 120:793-807, 2019. doi:10.1016/j. ymssp. 2018.11.009.

[14] D.L. Powers. Boundary Value Problems and Partial Differential Equations. Academic Press, 1972.

[15] M. Schatzman and M. Bercovier. Numerical approximation of a wave equation with unilateral constraints. Mathematics of Computation, 53(187):55-79, 1989. oai:hal-01295436 ə. doi:10.1090/ S0025-5718-1989-0969491-5.

[16] F. Schreyer and R.I. Leine. A mixed shooting-harmonic balance method for unilaterally constrained mechanical systems. Archive of Mechanical Engineering, LXIII(2):297-314, 2016. doi:10.1515/ meceng-2016-0017. 
[17] S.W. Shaw. Invariant manifold representations of nonlinear modes of vibration. In G. Kerschen, editor, Modal Analysis of Nonlinear Mechanical Systems, volume 555, pages 47-74. Springer, 2014. doi:10.1007/ 978-3-7091-1791-0_2.

[18] H. Tao and J. Gibert. Periodic orbits of a conservative 2-DOF vibro-impact system by piecewise continuation: bifurcations and fractals. Nonlinear Dynamics, 95:2963-2993, 2019. doi:10.1007/ s11071-018-04734-4.

[19] A. Thorin and M. Legrand. Nonsmooth modal analysis: From the discrete to the continuous settings. In $A d-$ vanced Topics in Nonsmooth Dynamics: Transactions of the European Network for Nonsmooth Dynamics, pages 191-234. Springer, 2018. oai:hal-01771849 ə. doi:10.1007/978-3-319-75972-2_5.

[20] D. Urman and M. Legrand. Nonlinear modal analysis of the bar in unilateral contact via analytical weaksolutions to the wave equation. In Proceedings of the 27th CANCAM, 2019. oai:hal-02151373 2.

[21] V.A. Yastrebov. Numerical Methods in Contact Mechanics. John Wiley \& Sons, 2013. doi:10.1002/ 9781118647974.

[22] C. Yoong. Nonsmooth modal analysis of a finite linear elastic bar subject to unilateral contact constraint. PhD thesis, McGill University, 2018. [pdf].

[23] C. Yoong, A. Thorin, and M. Legrand. Nonsmooth modal analysis of an elastic bar subject to a unilateral contact constraint. Nonlinear Dynamics, 91(4):2453-2476, 2018. oai:hal-01471341 ๑. doi:10.1007/ s11071-017-4025-9. 